\title{
How Can the Modified Dispersion Relation Affect Friedmann Equations?
}

\author{
A. S. Sefiedgar \\ Department of Physics, Faculty of Basic Sciences, University of Mazandaran, P.O. Box 47416-95447, Babolsar, Iran \\ Correspondence should be addressed to A. S. Sefiedgar; a.sefiedgar@umz.ac.ir
}

Received 20 January 2014; Accepted 15 March 2014; Published 1 April 2014

Academic Editor: Frank Filthaut

Copyright (C) 2014 A. S. Sefiedgar. This is an open access article distributed under the Creative Commons Attribution License, which permits unrestricted use, distribution, and reproduction in any medium, provided the original work is properly cited. The publication of this article was funded by SCOAP ${ }^{3}$.

\begin{abstract}
The appearance of the quantum gravitational effects in a very high energy regime necessitates some corrections to the thermodynamics of Friedmann-Robertson-Walker (FRW) universe. The modified dispersion relation (MDR) as a phenomenological approach to investigate the high energy physics provides a perturbation framework upon which the FRW universe thermodynamics can be corrected. In this letter, we obtain the corrected entropy-area relation of the apparent horizon of FRW universe by utilizing the extra dimensional form of MDR, leading to the modification of Friedmann equations. The influence of MDR on the Friedmann equations provides a good insight into the understanding of the FRW universe dynamics in the final quantum gravity theory.
\end{abstract}

\section{Introduction}

Nowadays a black hole is known as a thermodynamical object which obeys the four laws of thermodynamics. Using the entropy-area relation and the temperature relation of the black hole, the first law of thermodynamics can reproduce the gravitational field equations [1]. On the other hand, the Hawking radiation at the event horizon of the black hole, as a feature of Einstein gravity, is a quantum mechanics effect [2-5]. Hence one may find a deep connection between three seemingly different branches of science: thermodynamics, gravity, and quantum mechanics in the black holes. Recently, this connection has also been investigated extensively for FRW universe [6]. After replacing the event horizon of a black hole by the apparent horizon of FRW universe, one may attribute an associated entropy, $S=A / 4 G$, and temperature, $T=1 / 2 \pi \widetilde{r}_{A}$, to FRW spacetimes. Note that $A, G$, and $\tilde{r}_{A}$ are the area of the apparent horizon, the gravitational constant, and the radius of the apparent horizon, respectively. If one applies the first law of thermodynamics to the apparent horizon of any finite dimensional FRW universe with any spatial curvature, the Friedmann equations can be derived [6]. In addition, one may see the effects of quantum gravity in the process of Hawking radiation at the apparent horizon of the FRW universe. Therefore, the FRW spacetimes are suitable examples of extreme quantum gravity regimes as well as the black holes and a complete quantum theory of gravity is needed to describe them.

Since a common feature of all promising candidates for quantum theory of gravity is the existence of a minimal observable length [7-13], the modified dispersion relation is particularly suitable for incorporating such a finite resolution of the spacetime in the theoretical framework of the standard model [14]. In other words, MDR is a suitable phenomenological approach to study the physics of quantum gravity. The modifications of the energy-momentum dispersion relation are particularly so interesting in the study of loop quantum gravity and models based on noncommutative geometry [14]. The MDR affects the physical systems in a very high energy regime by incorporating the quantum gravitational effects. Since MDR can yield to a deeper understanding of the ultimate quantum gravity proposal, we have investigated the 4-dimensional form of MDR in the context of the black holes thermodynamics in [15]. There, we have compared the results obtained from MDR with ones obtained from some other more reliable approaches such as string theory and loop quantum gravity. Demanding the same results in all approaches leads to imposing some constraints on the form of MDR. Moreover, one may be interested in the extra 
dimensional form of the modified dispersion relation introduced in [16] with the aim of furthering our understanding of the properties and structure of MDR. In this letter, we use the extra dimensional form of MDR obtained in the abovementioned studies where terms proportional to odd powers of energy are not present [14-16] and concentrate on the $(n+1)$-dimensional FRW universe. MDR may affect the thermodynamics of FRW universe and then lead to the modification of the Friedmann equations.

Previously, FRW universe had been considered to modify the Friedmann equations from the generalized uncertainty principle (GUP) in 4 dimensions [17] and higher dimensions [2]. Although the extra dimensional form of the generalized uncertainty principle as an equivalence face of the modified dispersion relation has been used frequently in the past [1820], the modified dispersion relation in extra dimensions is a new subject worth discussing $[14,16]$. In this work, we start by introducing the extra dimensional form of MDR used by us. Then we apply MDR to obtain the corrections to the entropy-area relation of FRW universe. Using the corrected entropy-area relation of the apparent horizon, the first law of thermodynamics may be cast to the modified Friedmann equations. The modified Friedmann equations can certainly result in a better understanding of FRW universe dynamics.

\section{The Modified Dispersion Relation in Extra Dimensions}

The modified dispersion relation in 4 dimensions can be written as [21]

$$
\begin{aligned}
(\vec{p})^{2}= & f\left(E, m ; L_{p}\right) \simeq E^{2}-\mu^{2}+\alpha_{1} L_{p} E^{3} \\
& +\alpha_{2} L_{p}^{2} E^{4}+\mathcal{O}\left(L_{p}^{3} E^{5}\right),
\end{aligned}
$$

where the function $f$ gives the exact dispersion relation and $L_{p}$ is the Planck length. The applicability of a Taylor-series expansion for $E \ll 1 / L_{p}$ has been assumed on the righthand side. The values of coefficients $\alpha_{i}$ may be different in different proposals of quantum gravity. The parameter $m$ is the rest energy of the particle and the parameter $\mu$ on the right-hand side is directly related to the rest energy, but $\mu \neq m$ if $\alpha_{i}$ 's do not all vanish. In the context of black holes studies, incorporating quantum gravitational effects forces us to modify the Bekenstein-Hawking formalism. Of course, MDR can provide a perturbation framework for such modifications. On the other hand, string theory and loop quantum gravity give the entropy-area relation of black holes as

$$
S=\frac{A}{4 L_{p}^{2}}+\rho \ln \frac{A}{L_{p}^{2}}+\mathcal{O}\left(\frac{L_{p}^{2}}{A}\right)
$$

where the assumption $A \gg L_{p}^{2}$ has been considered and $\rho$ may have different values in string theory and in loop quantum gravity [21-26]. Since the black hole thermodynamical solutions obtained by using string theory and loop quantum gravity are expected to be more reliable, these solutions could be considered as a test bed against which other solutions including the ones obtained via MDR should be compared [15]. With that in mind, the entropy of a black hole obtained using (1) is functionally different from (2) obtained within string theory and loop quantum gravity. To obtain a consistent black hole thermodynamics in both approaches, it is necessary to introduce constraints on the usual form of the MDR. As a result, the terms proportional to odd powers of energy should be ignored in the MDR formula [15]. Consequently, the 4-dimensional form of MDR can be considered as

$$
(\vec{p})^{2}=f\left(E, m ; L_{p}\right) \simeq E^{2}-\mu^{2}+\alpha L_{p}^{2} E^{4}+\mathcal{O}\left(L_{p}^{4} E^{6}\right) .
$$

Of course, the thermodynamics of black holes obtained via (3) is now consistent with the result given by (2).

Alternatively, one may be interested in using the extra dimensional form of MDR which is not studied enough yet. We have investigated the modified dispersion relation in theories with extra dimensions in our previous work [16]. We know that the generalized uncertainty principle plays the same role as MDR in quantum theories of gravity. In other words, the generalized uncertainty principle is an appropriate approach to investigate quantum gravity and implies the concept of the minimal observable length in the same way as MDR. In fact, there is a phenomenologically close relation between GUP and MDR. It seems that GUP and MDR are phenomenologically two (though seemingly different) faces of an underlying quantum gravity proposal. Because of that one may find the extra dimensional form of MDR by using the extra dimensional form of GUP which is known in literatures. In 4 dimensions, GUP and MDR both provide a framework to modify the quantities in the realm of quantum gravity and yield to the same functional modifications. Consequently, one may expect that they can also yield to the equivalent results in extra dimensions. Utilizing the extra dimensional form of GUP and insisting to derive the equivalent results from GUP and MDR, one may introduce the extra dimensional form of MDR [16]. According to [16], the extra dimensional form of MDR is functionally the same as the 4-dimensional one in (3). However, it is important to point that the Planck length in the extra dimensional form of MDR depends on the dimensionality of the spacetime to incorporate the effects of the existence of the extra dimensions.

As another point, we have shown in $[14,16,27]$ that the parameter $\alpha$ is a negative quantity of order one. There, a comparison was made between the results of two approaches, GUP and MDR, within the context of the black hole thermodynamics with that of string theory and loop quantum gravity. Demanding the same results in all approaches and considering the string theory and loop quantum gravity as more comprehensive and needing to have the positive minimal observable length lead to the negativity of the parameter $\alpha$. The sign of the parameter $\alpha$ may play an important role in the interpretation of the correction terms coming from MDR. In this work, we utilize the extra dimensional form of MDR to modify the thermodynamics of the $(n+1)$-dimensional FRW universe. 


\section{The FRW Universe Thermodynamics}

We consider an $(n+1)$-dimensional FRW universe, whose line element is given by

$$
d s^{2}=-d t^{2}+a^{2}(t)\left(\frac{d r^{2}}{1-k r^{2}}+r^{2} d \Omega_{n-1}^{2}\right),
$$

where $d \Omega_{n-1}^{2}$ denotes the line element of an $(n-1)$-dimensional unit radius sphere, $a(t)$ is the scale factor, and $k$ defines the curvature of the spatial section. Using the notation $\widetilde{r}=a r$, one can find the radius of the apparent horizon as

$$
\tilde{r}_{A}=\frac{1}{\sqrt{H^{2}+k / a^{2}}}
$$

where $H=\dot{a} / a=(d a / d t) / a$ is the Hubble parameter. The apparent horizon is a marginally trapped surface with vanishing expansion. We assume that the apparent horizon has an associated entropy and temperature as

$$
S=\frac{A}{4 G}, \quad T=\frac{1}{2 \pi \widetilde{r}_{A}},
$$

where $A=n \Omega_{n} \widetilde{r}_{A}^{n-1}$ is the apparent horizon area and $\Omega_{n}=$ $\pi^{n / 2} / \Gamma(n / 2+1)$ is the volume of an $n$-dimensional unit sphere.

Then it can be shown that the first law of thermodynamics

$$
d E=T d S
$$

reproduces the Friedmann equations [6].

In this work, we are interested in finding the modified Friedmann equations in a very high energy regime. To obtain the modified Friedmann equations, we start by deriving the corrections to the entropy of FRW universe via MDR. Let us consider the extra dimensional form of MDR as

$$
\begin{aligned}
(\vec{p})^{2}= & f\left(E, m ; L_{p}\right) \simeq E^{2}-\mu^{2}+\alpha L_{p}^{2} E^{4} \\
& +\alpha^{\prime} L_{p}^{4} E^{6}+\mathcal{O}\left(L_{p}^{6} E^{8}\right) .
\end{aligned}
$$

Differentiating (8) yields

$$
d p \simeq d E\left[1+\frac{3}{2} \alpha L_{p}^{2} E^{2}+\left(\frac{5}{2} \alpha^{\prime}-\frac{5}{8} \alpha^{2}\right) L_{p}^{4} E^{4}\right],
$$

where the rest mass has been neglected. Here we have considered only the terms up to the forth power of the Planck length, without loss of generality in conclusion. Some manipulations will then lead to

$$
d E \simeq d p\left[1-\frac{3}{2} \alpha L_{p}^{2} E^{2}+\left(-\frac{5}{2} \alpha^{\prime}+\frac{23}{8} \alpha^{2}\right) L_{p}^{4} E^{4}\right] .
$$

To first order corrections, assuming $E \simeq \delta E$, we may apply the standard uncertainty formulae, $\delta E \geq 1 / \delta x$ and $\delta p \geq 1 / \delta x$, to obtain $[14-16,27]$

$$
d E \geq \frac{1}{\delta x}\left[1-\frac{3}{2} \alpha L_{p}^{2} \frac{1}{\delta x^{2}}+\left(-\frac{5}{2} \alpha^{\prime}+\frac{23}{8} \alpha^{2}\right) L_{p}^{4} \frac{1}{\delta x^{4}}\right] .
$$

Within the standard model, one may only see the first term on the right-hand side of the above formula. The emergence of the other terms refers to the effects of MDR. One may write (11) as

$$
d E \geq \frac{1}{\delta x} \Phi\left(\delta x^{2}\right)
$$

where

$$
\Phi\left(\delta x^{2}\right)=\left[1-\frac{3}{2} \alpha L_{p}^{2} \frac{1}{\delta x^{2}}+\left(-\frac{5}{2} \alpha^{\prime}+\frac{23}{8} \alpha^{2}\right) L_{p}^{4} \frac{1}{\delta x^{4}}\right] .
$$

Let us now consider that a particle with energy $d E$ is absorbed or radiated via the apparent horizon. In the realm of the standard model where the effects of quantum gravity can be ignored, the energy of this particle may be identified by $d E \simeq \delta p$ (with $c=1$ ) [28]. Using the standard uncertainty principle, $\delta p \geq 1 / \delta x$, one may find from (6) and (7) that

$$
d A=\frac{4 G}{T} d E \simeq \frac{4 G}{T} \frac{1}{\delta x} .
$$

One may obviously conclude that incorporating the effects of MDR via the inclusion of $\Phi$ yields

$$
d A_{\mathrm{MDR}}=\frac{4 G}{T} d E \simeq \frac{4 G}{T} \frac{1}{\delta x} \Phi\left(\delta x^{2}\right) .
$$

Using (14), we can write

$$
d A_{\mathrm{MDR}} \simeq \Phi\left(\delta x^{2}\right) d A
$$

The position uncertainty of the particle which is crossing through the apparent horizon can be chosen as its Compton wavelength which has the order of the inverse of the Hawking temperature. Thus one can write [29-32]

$$
\delta x \simeq 2 \widetilde{r}_{A}=2\left(\frac{A}{n \Omega_{n}}\right)^{1 /(n-1)}
$$

where $\Omega_{n}$ is the volume of an $n$-dimensional unit sphere. Now it is possible to write $\Phi\left(\delta x^{2}\right)$ as a function of the area of the apparent horizon as follows:

$$
\begin{aligned}
\Phi(A)= & 1-\frac{3}{8} \alpha L_{p}^{2}\left(\frac{n \Omega_{n}}{A}\right)^{2 /(n-1)} \\
& +\left(-\frac{5}{32} \alpha^{\prime}+\frac{23}{128} \alpha^{2}\right) L_{p}^{4}\left(\frac{n \Omega_{n}}{A}\right)^{4 /(n-1)} .
\end{aligned}
$$

Substituting (18) into (16), one may find the area of the apparent horizon as

$$
A_{\mathrm{MDR}}=\int \Phi(A) d A .
$$

As a result, the corrected entropy of FRW universe can be derived as

$$
S_{\mathrm{MDR}}=\frac{A_{\mathrm{MDR}}}{4 G} .
$$


For $n=3$, the MDR-corrected entropy of the FRW universe will be

$$
\begin{aligned}
S_{\mathrm{MDR}}= & \frac{A}{4 G}-\frac{3}{8} \alpha L_{p}^{2}\left(\frac{3 \Omega_{3}}{4 G}\right) \ln \frac{A}{4 G} \\
& -\left(-\frac{5}{32} \alpha^{\prime}+\frac{23}{128} \alpha^{2}\right) L_{p}^{4}\left(\frac{3 \Omega_{3}}{4 G}\right)^{2} \frac{4 G}{A} .
\end{aligned}
$$

For $n=4$, the corrected entropy is

$$
\begin{aligned}
S_{\mathrm{MDR}}= & \frac{A}{4 G}-\frac{9}{2} \alpha L_{p}^{2}\left(\frac{4 \Omega_{4}}{4 G}\right)^{2 / 3}\left(\frac{A}{4 G}\right)^{1 / 3} \\
& -3\left(-\frac{5}{32} \alpha^{\prime}+\frac{23}{128} \alpha^{2}\right) L_{p}^{4}\left(\frac{4 \Omega_{4}}{4 G}\right)^{4 / 3}\left(\frac{4 G}{A}\right)^{1 / 3},
\end{aligned}
$$

and for $n=5$, the MDR-corrected entropy is

$$
\begin{aligned}
S_{\mathrm{MDR}}= & \frac{A}{4 G}-\frac{6}{8} \alpha L_{p}^{2}\left(\frac{5 \Omega_{5}}{4 G}\right)^{1 / 2}\left(\frac{A}{4 G}\right)^{1 / 2} \\
& +\left(-\frac{5}{32} \alpha^{\prime}+\frac{23}{128} \alpha^{2}\right) L_{p}^{4}\left(\frac{5 \Omega_{5}}{4 G}\right) \ln \frac{A}{4 G} .
\end{aligned}
$$

The entropy-area relation of FRW universe can be similarly derived for another dimensionality. Without loss of generality, we have considered the influence of the MDR on the entropy-area relation of the FRW universe only up to the forth power of the Planck length. The existence of the logarithmic correction term in the entropy-area relation of a FRW universe or a black hole has been so controversial in literatures $[2,14-17,33]$. In this work, it has been shown that the logarithmic correction term has appeared only for odd $n$ (even spacetime dimensions). In other words, if one tries to obtain the entropy-area relation for other dimensions, the emergence of the logarithmic correction term is certainly restricted to even dimensional universe. Moreover, one may see that the leading logarithmic correction term gives a positive contribution to the entropy of the 4-dimensional spacetime since the parameter $\alpha$ is a negative quantity of order one $[14,16,27]$. Since this particular term may be useful as a discriminator of the prospective fundamental theories, fixing it independent of the specific elements of any particular model of quantum gravity seems to be of importance. Therefore, MDR as a model independent concept is a convenient approach to provide the corrections to FRW universe entropy.

As another point, we can refer to the absence of the fractional powers of $A$ and $A^{-1}$ in the entropy-area relation of the 4-dimensional FRW universe in our approach. In 4 dimensions, the entropy-area relation obtained here is functionally consistent with the results coming from the GUP in [2], whereas it is different from the results coming from the GUP in [17]. It seems that the MDR which is applied by us may be another face of the GUP version without the linear term of the Planck length in [2].

Anyway, the quantum gravity effects are so important in the early universe. The appearance of the correction terms in the entropy-area relation, coming from MDR, plays an important role in studying the FRW universe in the Planck time. In addition, one may be interested in finding the modified Friedmann equations within MDR. Using the MDRcorrected entropy of FRW universe, the first law of thermodynamics can reproduce the modified Friedmann equations.

\section{The Modified Friedmann Equations}

In an $(n+1)$-dimensional FRW universe with the line element given by (4), we may suppose that the apparent horizon has an associated entropy, $S=A / 4 G$, and temperature, $T=1 / 2 \pi \widetilde{r}_{A}$. It has been confirmed that the first law of thermodynamics, $d E=T d S$ can reproduce the Friedmann equations [6]

$$
\begin{gathered}
\dot{H}-\frac{k}{a^{2}}=-\frac{8 \pi G}{n-1}(\rho+P), \\
H^{2}+\frac{k}{a^{2}}=\frac{16 \pi G}{n(n-1)} \rho,
\end{gathered}
$$

where $\rho$ and $P$ are the energy density and pressure of the cosmic fluid, respectively. $d E=d(\rho V)$ is the energy flow pass through the apparent horizon. To derive the Friedmann equations, the continuity equation has been used.

It is important to point that the modification to the entropy-area relation leads to the modification to Friedmann equations $[2,17,34]$. In this work, we have applied MDR to correct the entropy-area relation of FRW universe. Now we can write the first law of thermodynamics as

$$
d E=T d S_{\mathrm{MDR}}
$$

where $T=1 / 2 \pi \widetilde{r}_{A}$ and $d E=A(\rho+P) H \widetilde{r}_{A} d t$. By some manipulations, the modified Friedmann equations can be written as [2]

$$
\begin{gathered}
\left(\dot{H}-\frac{k}{a^{2}}\right) \Phi(A)=-\frac{8 \pi G}{n-1}(\rho+P), \\
\frac{8 \pi G}{n} \rho=-\int \Phi(A)\left(\frac{A}{n \Omega_{n}}\right)^{-2 /(n-1)} \frac{d A}{A} .
\end{gathered}
$$

The function $\Phi(A)$ has been introduced in the previous section and shows the effects of MDR. Finding $\Phi(A)$ from (18) and substituting the result into (26) lead to the corrected Friedmann equations.

For $n=3$, the modified Friedmann equations can be derived as

$$
\begin{aligned}
-\frac{8 \pi G}{2}(\rho+P)= & \left(\dot{H}-\frac{k}{a^{2}}\right) \\
& \times\left[1-\frac{3}{8} \alpha L_{p}^{2}\left(\frac{3 \Omega_{3}}{A}\right)\right. \\
& \left.+\left(-\frac{5}{32} \alpha^{\prime}+\frac{23}{128} \alpha^{2}\right) L_{p}^{4}\left(\frac{3 \Omega_{3}}{A}\right)^{2}\right],
\end{aligned}
$$




$$
\begin{aligned}
\frac{8 \pi G}{3} \rho= & \frac{3 \Omega_{3}}{A}-\frac{3}{16} \alpha L_{p}^{2}\left(\frac{3 \Omega_{3}}{A}\right)^{2} \\
& +\left(-\frac{5}{96} \alpha^{\prime}+\frac{23}{384} \alpha^{2}\right) L_{p}^{4}\left(\frac{3 \Omega_{3}}{A}\right)^{3} .
\end{aligned}
$$

For $n=4$, we may have

$$
\begin{aligned}
-\frac{8 \pi G}{3}(\rho+P)= & \left(\dot{H}-\frac{k}{a^{2}}\right) \\
& \times\left[1-\frac{3}{8} \alpha L_{p}^{2}\left(\frac{4 \Omega_{4}}{A}\right)^{2 / 3}\right. \\
& \left.+\left(-\frac{5}{32} \alpha^{\prime}+\frac{23}{128} \alpha^{2}\right) L_{p}^{4}\left(\frac{4 \Omega_{4}}{A}\right)^{4 / 3}\right], \\
\frac{8 \pi G}{4} \rho= & \left.\frac{3}{2}\left(\frac{4 \Omega_{4}}{A}\right)^{2 / 3}-\frac{9}{32} \alpha L_{p}^{2}\left(\frac{4 \Omega_{4}}{A}\right)^{4 / 3}\right] \\
& +\left(-\frac{5}{64} \alpha^{\prime}+\frac{23}{256} \alpha^{2}\right) L_{p}^{4}\left(\frac{4 \Omega_{4}}{A}\right)^{2} .
\end{aligned}
$$

For $n=5$, we have

$$
\begin{aligned}
-\frac{8 \pi G}{4}(\rho+P)= & \left(\dot{H}-\frac{k}{a^{2}}\right) \\
& \times\left[1-\frac{3}{8} \alpha L_{p}^{2}\left(\frac{5 \Omega_{5}}{A}\right)^{1 / 2}\right. \\
& \left.+\left(-\frac{5}{32} \alpha^{\prime}+\frac{23}{128} \alpha^{2}\right) L_{p}^{4}\left(\frac{5 \Omega_{5}}{A}\right)\right], \\
\frac{8 \pi G}{5} \rho= & 2\left(\frac{5 \Omega_{5}}{A}\right)^{1 / 2}-\frac{3}{8} \alpha L_{p}^{2}\left(\frac{5 \Omega_{5}}{A}\right) \\
& +\left(-\frac{10}{96} \alpha^{\prime}+\frac{46}{384} \alpha^{2}\right) L_{p}^{4}\left(\frac{5 \Omega_{5}}{A}\right)^{3 / 2} .
\end{aligned}
$$

Here we have kept only the terms up to the forth power of the Planck length. The Friedmann equations for another dimensionality can be derived in a similar way. Here MDR has provided a useful framework to incorporate the quantum gravitational effects in a very high energy regime. Of course, understanding more about Friedmann equations leads to more accurate dynamics for the universe. Realizing the dynamics of the FRW universe in the era of Planck time can be helpful in paving the way for studying the whole evolutionary history of the universe.

\section{Conclusions}

Finding the whole evolution history of the FRW universe is one of the most important goals in cosmology. To reach this goal, one may be interested in finding the dynamics of the FRW universe in the era of Planck time as an important part of this history. Since the early universe experiences a very high energy regime, the effects of quantum gravity should not be forgotten there. In this letter, the modified dispersion relation as an appropriate phenomenological approach to study the quantum gravitational effects has been used to study the FRW universe. We have obtained the corrections to the entropy of a FRW universe via the extra dimensional form of MDR. We have found that the logarithmic correction term, whose existence is still somewhat mysterious, may only emerge in a FRW universe with even dimensions (odd $n$ 's). In other words, the existence of the logarithmic correction term depends on the spacetime dimensions. Insisting on the existence of the logarithmic correction term in the entropy of a FRW universe restricts the spacetime dimensions to be even. This fact can provide a constraint on the ultimate quantum gravity theory. As another point, according to the negativity of parameter $\alpha$, one can conclude that the leading logarithmic correction term has a positive contribution to the entropy of the apparent horizon of the 4-dimensional FRW universe. Utilizing the corrected entropy-area relation, the first law of thermodynamics can reproduce the modified Friedmann equations. The corrections to the Friedmann equations can certainly lead to a more accurate dynamics and hence a better understanding of the evolution history of the FRW universe.

\section{Conflict of Interests}

The author declares that there is no conflict of interests regarding the publication of this paper.

\section{References}

[1] T. Jacobson, "Thermodynamics of spacetime: the Einstein equation of state," Physical Review Letters, vol. 75, no. 7, pp. 1260$1263,1995$.

[2] T. Zhu, J.-R. Ren, and M.-F. Li, "Influence of generalized and extended uncertainty principle on thermodynamics of FRW universe," Physics Letters B, vol. 674, no. 3, pp. 204-209, 2009.

[3] S. W. Hawking, "Particle creation by black holes," Communications in Mathematical Physics, vol. 43, no. 3, pp. 199-220, 1975.

[4] S. W. Hawking, "Breakdown of predictability in gravitational collapse," Physical Review D, vol. 14, no. 10, pp. 2460-2473, 1976.

[5] M. K. Parikh and F. Wilczek, "Hawking radiation as tunneling," Physical Review Letters, vol. 85, no. 24, pp. 5042-5045, 2000.

[6] R.-G. Cai and S. P. Kim, "First law of thermodynamics and Friedmann equations of Friedmann-Robertson-Walker universe," Journal of High Energy Physics, no. 2, article 050, 2005.

[7] D. J. Gross and P. F. Mende, "String theory beyond the Planck scale," Nuclear Physics B, vol. 303, no. 3, pp. 407-454, 1988.

[8] K. Konishi, G. Paffuti, and P. Provero, "Minimum physical length and the generalized uncertainty principle in string theory," Physics Letters B, vol. 234, no. 3, pp. 276-284, 1990.

[9] D. Amati, M. Ciafaloni, and G. Veneziano, "Can spacetime be probed below the string size?” Physics Letters B, vol. 216, no. 1-2, pp. 41-47, 1989.

[10] C. Rovelli, "Loop quantum gravity," Living Reviews in Relativity, vol. 1, article 1, 1998.

[11] A. Ashtekar and J. Lewandowski, "Background independent quantum gravity: a status report," Classical and Quantum Gravity, vol. 21, no. 15, pp. R53-R152, 2004. 
[12] M. R. Douglas and N. A. Nekrasov, "Noncommutative field theory," Reviews of Modern Physics, vol. 73, p. 977, 2001.

[13] F. Girelli, E. R. Livine, and D. Oriti, "Deformed special relativity as an effective flat limit of quantum gravity," Nuclear Physics B, vol. 708, pp. 411-433, 2005.

[14] A. S. Sefiedgar and H. R. Sepangi, "Corrections to the CardyVerlinde formula from the modified dispersion relation in extra dimensions," Physics Letters B, vol. 706, no. 4-5, pp. 431-435, 2012.

[15] K. Nozari and A. S. Sefidgar, "Comparison of approaches to quantum correction of black hole thermodynamics," Physics Letters B, vol. 635, no. 2-3, pp. 156-160, 2006.

[16] A. S. Sefiedgar, K. Nozari, and H. R. Sepangi, "Modified dispersion relations in extra dimensions," Physics Letters B, vol. 696, no. 1-2, pp. 119-123, 2011.

[17] B. Majumder, "The generalized uncertainty principle and the Friedmann equations," Astrophysics and Space Science, vol. 336, no. 2, pp. 331-335, 2011.

[18] B. Bolen and M. Cavaglià, "(Anti-)de Sitter black hole thermodynamics and the generalized uncertainty principle," General Relativity and Gravitation, vol. 37, no. 7, pp. 1255-1262, 2005.

[19] A. Kempf, G. Mangano, and R. B. Mann, "Hilbert space representation of the minimal length uncertainty relation," Physical Review D, vol. 52, no. 2, pp. 1108-1118, 1995.

[20] A. Kempf, "Non-pointlike particles in harmonic oscillators," Journal of Physics, vol. 30, no. 6, pp. 2093-2101, 1997.

[21] G. Amelino-Camelia, M. Arzano, Y. Ling, and G. Mandanici, "Black-hole thermodynamics with modified dispersion relations and generalized uncertainty principles," Classical and Quantum Gravity, vol. 23, no. 7, pp. 2585-2606, 2006.

[22] G. Amelino-Camelia, M. Arzano, and A. Procaccini, "A glimpse at the flat-spacetime limit of quantum gravity using the Bekenstein argument in reverse," International Journal of Modern Physics D, vol. 13, no. 10, p. 2337, 2004.

[23] G. Amelino-Camelia, "A phenomenological description of space-time noise in quantum gravity," Nature, vol. 410, pp. 10651067, 2001.

[24] J. Magueijo and L. Smolin, "Generalized Lorentz invariance with an invariant energy scale," Physical Review D, vol. 67, Article ID 044017, 2003.

[25] G. Amelino-Camelia, "Relativity in spacetimes with short-distance structure governed by an observer-independent (Planckian) length scale," International Journal of Modern Physics D, vol. 11, no. 1, p. 35, 2002.

[26] Y. Ling, B. Hu, and X. Li, "Modified dispersion relations and black hole physics," Physical Review D, vol. 73, Article ID 087702, 2006.

[27] A. S. Sefiedgar and H. R. Sepangi, "Brane-world black hole entropy from modified dispersion relations," Physics Letters B, vol. 692, pp. 281-285, 2010.

[28] M.-I. Park, "The generalized uncertainty principle in (A)dS space and the modification of Hawking temperature from the minimal length," Physics Letters B, vol. 659, no. 3, pp. 698-702, 2008.

[29] K. Nozari and A. S. Sefidgar, "On the existence of the logarithmic correction term in black hole entropy-area relation," General Relativity and Gravitation, vol. 39, no. 4, pp. 501-509, 2007.

[30] A. J. M. Medved and E. C. Vagenas, "When conceptual worlds collide: the generalized uncertainty principle and the Bekenstein-Hawking entropy," Physical Review D, vol. 70, Article ID 124021, 2004.
[31] R. Zhao and S. L. Zhang, "Generalized uncertainty principle and black hole entropy," Physics Letters B, vol. 641, p. 208, 2006.

[32] H. X. Zhao, H. F. Li, S. Q. Hu, and R. Zhao, "Generalized uncertainty principle and black hole entropy of higher-dimensional de sitter spacetime," Communications in Theoretical Physics, vol. 48, no. 3, p. 465, 2007.

[33] A. J. M. Medved, "A comment on black hole entropy or does nature abhor a logarithm?” Classical and Quantum Gravity, vol. 22, no. 1, pp. 133-142, 2005.

[34] R.-G. Cai, L.-M. Cao, and Y.-P. Hu, "Corrected entropy-area relation and modified Friedmann equations," Journal of High Energy Physics, no. 8, article 090, 2008. 

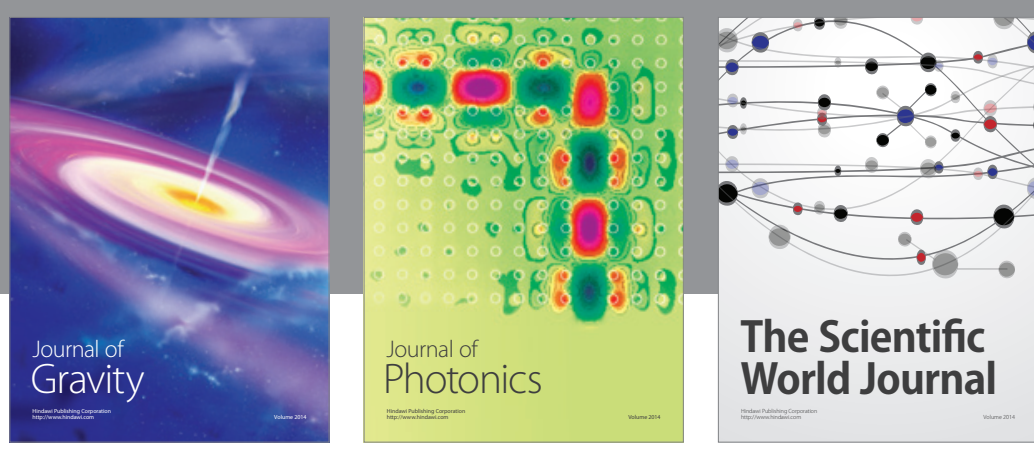

The Scientific World Journal
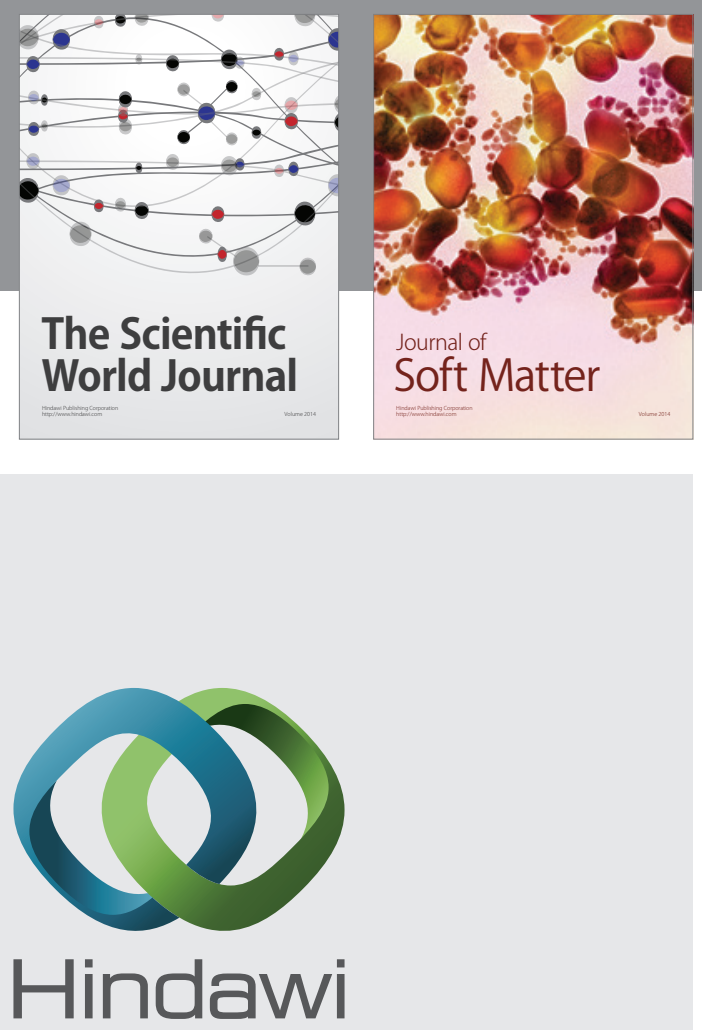

Submit your manuscripts at

http://www.hindawi.com

nternational Journal of

Statistical Mechanics
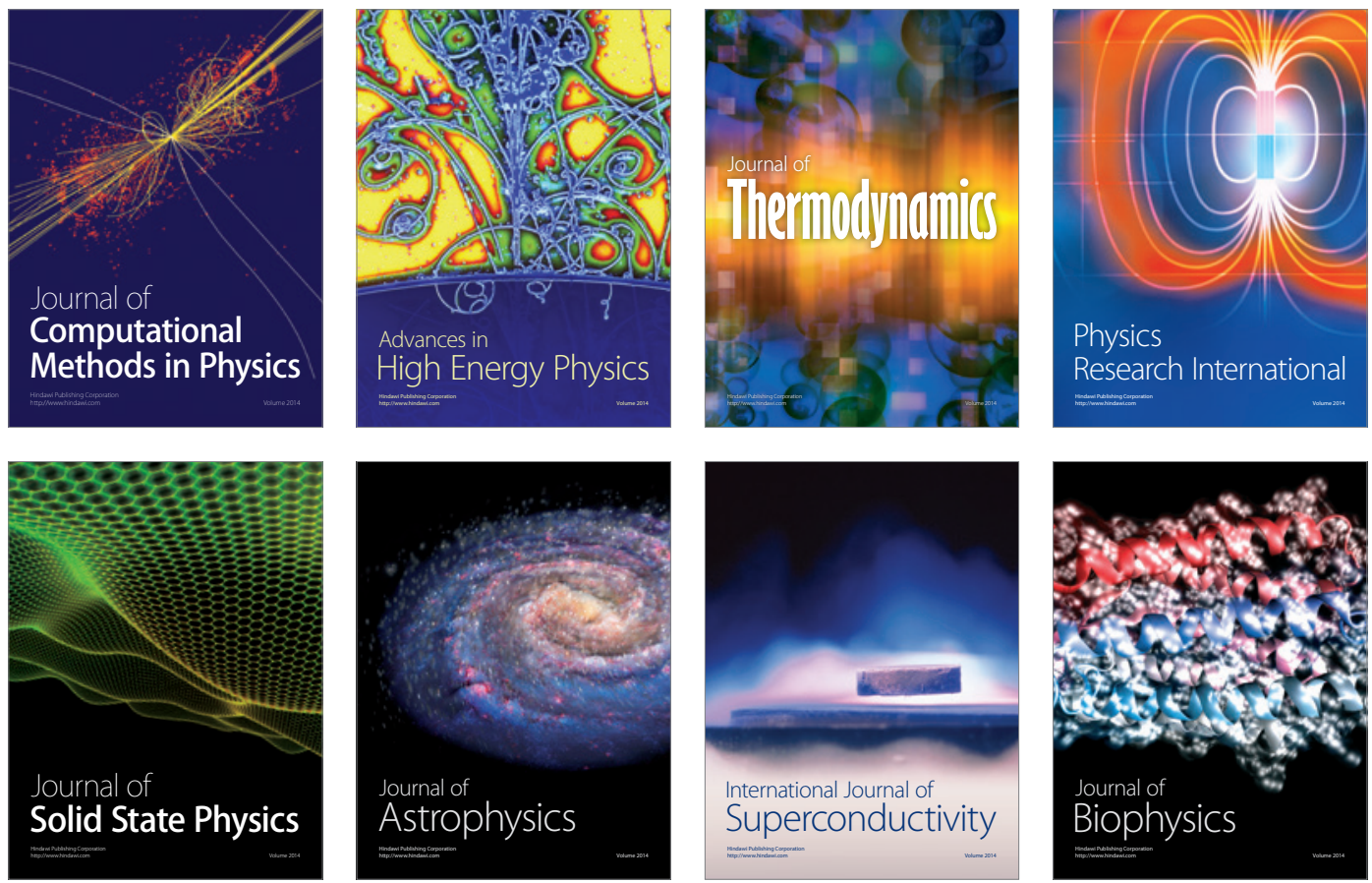
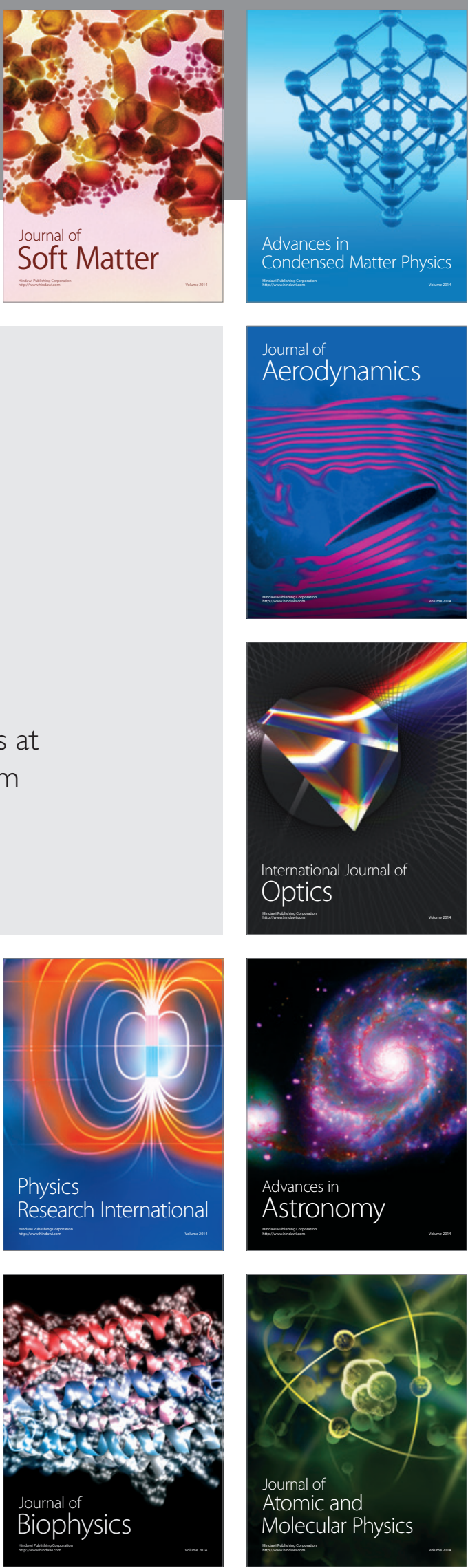